\title{
Factors Associated with Stunting in School Children of an Urban Community in Cameroon
}

\author{
Georges Pius Kamsu Moyo ${ }^{1, \text { *, Ivan Fred Kamsi Djomkam² }}$ \\ ${ }^{1}$ Faculty of Medicine and Biomedical Sciences, University of Yaounde I, Yaounde, Cameroon \\ ${ }^{2}$ Soa, District Hospital, Yaounde, Cameroon
}

Email address:

kamsuzicfried@yahoo.fr (G. P. K. Moyo)

${ }^{*}$ Corresponding author

\section{To cite this article:}

Georges Pius Kamsu Moyo, Ivan Fred Kamsi Djomkam. Factors Associated with Stunting in School Children of an Urban Community in Cameroon. American Journal of Pediatrics. Vol. 6, No. 2, 2020, pp. 121-124. doi: 10.11648/j.ajp.20200602.20

Received: February 7, 2020; Accepted: February 27, 2020; Published: March 17, 2020

\begin{abstract}
Background: Stunting is a condition that occurs as a result of a defective growth process. It may remain unnoticed in some children, with the diagnosis made as late as during adolescence. The accurate diagnosis of stunting is mainly done by physical examination, including the assessment of anthropometric parameters, while etiological explorations may require deeper and more complex investigations such as radiological and hormonal or endocrinal analyses. The various etiologies described in the literature may vary from nutritional, hormonal, pathological to psychological factors. A number of determinants of stunting in children have equally been documented, but they may vary from one context to another according to environmental factors. The effective management of stunting generally relies on the treatment of the underlying causes and the prevention of risk factors. Our objective was to determine the factors associated with stunting in school children living in an urban community of Yaounde, Cameroon. Methods: An analytic cross-sectional study was carried out on primary and secondary school children aged from 8 to 15 years, between January and June 2015. Bivariate and multivariate analyses were made to identify risk factors and eventually predictive factors. Results: The study involved 1442 children of which 79 presented stature delay. After bivariate analysis, the risk factors associated with stunting were: the presence of chronic pathological conditions $(\mathrm{p}=0.03)$, low socioeconomic level $(\mathrm{p}=0.0022)$, poor or limited diet $(\mathrm{p}=0.03)$, delayed puberty $(\mathrm{p}=0)$, and children "living with their mothers only" $(\mathrm{p}=0.02)$. After multivariate analysis, low socioeconomic level $(\mathrm{OR}=4.2 ; \mathrm{CI}=1.7$ 10.3), children "living with their mother only" $(\mathrm{OR}=0.2 ; \mathrm{CI}=0.6-0.8)$, and delayed puberty $(\mathrm{OR}=12.6 ; \mathrm{CI}=4.9-31.8)$ persisted as independent predictive factors. Conclusion: Common risk factors such as chronic pathologies, low socioeconomic level, poor or limited diet and delayed puberty which are generally incriminated by various researches were verified in this survey. Delayed puberty was the most strongly associated factor, followed by "low socioeconomic level" and children "living with their mothers only", which appeared as predictive factors as well. Nevertheless, children "living with their mothers only" were protected from stunting. Therefore, improvement of socioeconomic standards, food quality, prevention of chronic pathologies and delayed puberty may help to reduce stunting in such children. While mothers' presence for nurturing and affective attention is necessary.
\end{abstract}

Keywords: Stunting, School Children, Cameroon

\section{Introduction}

The linear growth of a child may be described as a dynamic process that extends from birth to adult age through childhood and adolescence, under the influence of genetic, nutritional, hormonal and psycho-affective factors [1, 2]. It comprises various stages with specific characteristics such as growth rate and maturation $[1,2]$. Stunting results from a defective growth and is a common presenting complaints during pediatric consultations. it may be the first sign of a pathology susceptible to jeopardize the vital or functional prognosis of a child. The main causes of a defective growth found in the literature are poor nutrition, chronic diseases, skeletal, or hormonal abnormalities [2]. Global statistics show $25 \%$ of primary school children suffered stature growth 
delay around the year 2000 [3]. The situation was more pronounced in low-income countries where over $56 \%$ of children were affected by stunting [4]. In Africa, the prevalence of stature growth delay increases with age and may be associated with cognitive impairments, inadequate academic performances and school dropping [5-7]. As far as Cameroon is concerned, the National Institute of Statistics reported the childhood incidence of stunting at $29 \%$ in 1998 [8]. However, few studies give account of factors associated with short stature in children in our context. This survey in the urban community of Yaoundé aims to identify the various determinants of stunting in school children.

\section{Methodology}

An analytic cross-sectional study was carried out in primary and secondary schools of the urban community of Yaoundé in Cameroon. For six months, from January to June 2015 , children aged 8 to 15 years, registered in selected schools and whose parents' authorizations were obtained, were included in the study. Random cluster sampling was done with primary statistical unit being the school (cluster) and the secondary statistical unit being the student. School selection was done from a list of educational institutions obtained from regional delegations of secondary and basic educations for the year 2015. The draw was conducted so as to have equal probability for each institution. As such, 10 schools were selected out of 1471 . The minimal sample size estimated was 1325 children with a confidence interval at $95 \%$ and $5 \%$ precision. The number of students who took part in the study was 1442. The children enrolled were submitted to a data collection sheet. The enquiries comprised demographic indicators, health status, socioeconomic level, nutritional assessment, psycho-environmental investigation and anthropometric measurements. The collected data was then transferred into a software-conceived database with EpiData and analyzed using STATA version 12.0. Bi variate analysis was done with Student's test or $\mathrm{Chi}^{2}$ according to the variable. Fischer's test served for analyzing small qualitative variables. Multivariate logistic regression was done to identify independent predictive factors associated with short stature.

\section{Results}

\subsection{General Characteristics}

We worked on a sample of 1442 children. The mean age was 12 years (IQR: 11-13ans). Fifty-one point nine percent $(51.9 \%)$ of children with stunting were of female sex. The majority of children with short stature were from families with average socioeconomic level (62\%), among which $62 \%$ had limited or poor diet while $28 \%$ had good diet according to the World Food Program (WFP). Ninety-six point two percent $(96.2 \%)$ of children with short stature had normal Body Mass Index (-1DS $<$ BMI $<1 \mathrm{DS})$ and $4.8 \%$ of them being girls with overweight $(+1 \mathrm{DS}<\mathrm{BMI}<+2 \mathrm{DS})$. Thirty- three percent $(33 \%)$ of children with stature growth delay had abnormal body segments. Delayed puberty according to Tarner's classification was found in $12.7 \%$ of children, among whom $3 \%$ reported having chronic kidney disease, $1.5 \%$ were affected with heart disease and sickle cell anemia occurred in $0.5 \%$. In $49.3 \%$ of children with stunting, no etiology was identified.

\subsection{Risk Factors}

Bivariate analysis showed relationships between chronic pathological conditions and stunting $(\mathrm{p}=0.03)$, as well as low socioeconomic level $(\mathrm{p}=0.0022)$, poor or limited diet $(\mathrm{p}=0.03)$, delayed puberty $(\mathrm{p}=0)$ and children "living with their mothers only" $(p=0.02)$. After multivariate analysis, low socioeconomic level $(\mathrm{p}=0.02)$, children "living with their mothers only" $(p=0.02)$, and delayed puberty $(p=0)$ persisted as predictive factors associated with stature growth delay. Table 1 below summarizes the various existing relationships after multivariate analysis.

\subsection{Predictive Factors}

Table 1. Multivariate analysis by logistic regression of factors associated with stunting.

\begin{tabular}{llll}
\hline Multivariate analysis & OR & CI 95\% & P \\
\hline SOCIOECONOMIC LEVEL & & & \\
High & 1.0 & & \\
Average & 1,1 & $0,6-2,1$ & 0,8 \\
Low & 4,2 & $1,7-10,3$ & 0,02 \\
DIET & & & \\
Good & 1.0 & & 0,3 \\
Limit & 1,4 & $0,7-2,6$ & 0,3 \\
Poor & 1,52 & $0,7-3,2$ & \\
PERSONS LIVING WITH CHILD & & & 0,02 \\
Other than the Single mother & 1.0 & & \\
Mother Only & 0,2 & $0,6-0,8$ & \\
CHRONIC DISEASE & & & 0,5 \\
Absence & 1.0 & & \\
Presence & 1,5 & $0,4-5,5$ & \\
DELAYED PUBERTY & & & \\
Absence & 1.0 & & \\
Presence & 12,6 & $4,9-31,8$ & 0 \\
\hline
\end{tabular}

\section{Discussion}

A relationship between stunting and low socioeconomic level was found in this survey, and is consistent with other studies conducted in the African continent, showing that adolescents from families with medium and high socioeconomic level are less prone to short stature, compared to those from families with low socioeconomic level $[9,10]$. This seems coherent as socioeconomic wellbeing may improve nutritional quality and gear stature development. Findings from a number of researches conducted in our context are in support of the hypothesis which suggest that stunting is particularly common in people with low income, insufficient access to drinking water and deprived of adequate health education $[11,12]$. however, the classification of socioeconomic level takes into consideration 
the income, the family size, the type of housing, and the level of parents' education, which are susceptible to influence though indirectly, the growth of children [9, 12]. These associations have equally been highlighted in numerous studies worldwide [9-12].

Poor or limited diet equally appeared to be a risk factor associated with the onset of short stature in this series. In effect, many studies reported insufficient dietary factors as a major determinant of growth failure [13, 14]. According to some researchers, food diversification is a key determinant of growth in children aged between 6-16 years [5]. Moreover, stature growth delay in children might be a long-term indicator of chronic undernourishment [13-15]. Given that the limitation of economic resources affects the accessibility to quality nutrition, low income may therefore lead to nutritional imbalances, malnutrition and hence stature growth delay $[10,11,13,14]$.

Stunting was also found to be associated with chronic pathological conditions in this survey. This could be due to the fact that chronic diseases lead to increased nutrients need and wasting, which if not provided may impact the growth process [1, 2, 15-17]. Therefore, chronic pathologies such as sickle cell anemia, diabetes, HIV-AIDS, heart and renal failure which might be quite prevalent and aggressive in African children may further increase the incidence of stunting in children.

Delayed puberty in this survey was the most strongly associated factor with stunting, and this is consistent with results from numerous studies [1-4]. In effect, sex hormones such as testosterone and estrogen play a key role in the growth process, especially during puberty when their secretion reach the climax. Nevertheless, this physiological process could be disrupted by delayed puberty or hormonal insufficiencies [1-4].

In this survey, children "living with their mothers only" seemed less affected by stunting. As such, the mother's presence beside the growing child appeared to be a protective factor preventing the onset of stunting. This could be due to the fact that mothers might be more affective and caring, especially in the absence of fathers, with favorable repercussions on children nutrition, emotional status and hence the growth rate. However, such a relationship had not been demonstrated before and so further investigations may be required for more understanding.

\section{Conclusion}

Common risk factors such as chronic pathologies, low socioeconomic level, poor or limited diet and delayed puberty which are generally incriminated by various studies were verified in this survey. Delayed puberty was the most strongly associated factor, followed by "low socioeconomic level" and children "living with their mothers only", which appeared as predictive factors as well. Nevertheless, children "living with their mothers only" were protected from stunting. Therefore, improvement of socioeconomic standards, food quality, the prevention of chronic pathologies and delayed puberty may help to prevent stunting. While mothers' presence for nurturing and affective attention is necessary.

\section{Author Contributions}

Authors participated in all steps of the study.

\section{Conflict of Interest}

The authors declare that they have no competing interest.

\section{Ethical Approval}

The study was approved by the Institutional Ethics Committee.

\section{Acknowledgements}

All collaborators to the project.

\section{References}

[1] Albalawi1 NAS, Alsabah1 BAB, Alrefaei AY, Alatawi AMS, Albalawi1 MS, Al-Enazi1 ZMMM et al. Short Stature in Children. Egypt J Hosp.2018; 70 (2): 228-233.

[2] Saengkaew T, McNeil E, Jaruratanasirikul S. Etiologies of short stature in a pediatric endocrine clinic in Southern Thailand. J Pediatr Endocrinol Metab 2017; 30 (12).

[3] El Hioui, Ahami A, Aboussaleh Y, Rusinek S. Staturoponderal deficit in studests from a Moroccan school. Bull. Soc. Pharm. Bordeaux 2008; 147: 61-7.

[4] Alharthi A. Idiopathic Short Stature in Children: A Hospital Based Study. J Med Sci. 2016; 16 (3): 56-61.

[5] Sbaibi R, Aboussaleh Y. An explorative study of the staturoponderal status of secondary school children in the rural community of Sidi El Kamel in North-west Marocco. Anthropo 2011; 24: 61-6.

[6] Zayed AA, Beano AM, Haddadin FI, Radwan SS, Allauzy SA, Alkhayyat MM et al. Prevalence of short stature, underweight, overweight, and obesity among school children in Jordan. BMC Public Health.2016; 16 (1): 1040.

[7] Berhe K, Seid O, Geremariam Y, Berhe A, Etsay N. Risk factors of stunting (chronic undernourishment) of children aged 6 to 24 moths I Mekelle City, Tigray region, North Ethiopia: an unmatched case-control study. PloS OnE.2019; 14 (6): e0217736.

[8] National Institute of Statistics of Cameroon. Health and demographic survey with multiple indicators. 2011.

[9] Jafari-Adli S, Qorbani M, Heshmat R, Ranjbar SH, Taheri E, et al. Association of short stature with life satisfaction and self-rated health in children and adolescents: the CASPIANIV study. J Pediatr Endocrinol Metab 2016; 29: 1299-306.

[10] Leonie N, Urban J, Nouedoui U, Hans S, Lena S. Socioeconomic and gender differences in adolescents' nutritional status in urban Cameroon, Africa. Nutritional research 2009; 29: 312-13. 
[11] Bernard O, Pagezy H, Bley D. Nutritional and environment pathogen of children in a forest population of southCameroon. Reports and memories of the Society of Anthropology of Paris. 2001; 13 (1-2).

[12] Sbaibi R, Aboussaleh Y, Achouri I, Ahami A, Ateillal K. Exploration of relationships between the staturo-ponderal status and certain socioeconomic factors in secondary school students in the rural community of Sidi El Kamel (north-west marocco). Anthropo 2014; 31: 9-16.

[13] WHO. Turning the tide of malnutrition responding to the challenge of the 21 st century (who/NHD/007), Geneve.2000.

[14] Joint Malnutrition dataset from UNICEF, World Bank and WHO. http://data.unicef.org/nutrition/malnutrition.html. 2016.
[15] Das S, Gulshan J. Different forms of malnutrition among under five children in Bangladesch: a cross sectional study on prevalence and determinants. BMC nutr. 2017; 3 (1): 1.

[16] Villanueva C, Argente J. Pathology or normal variant: what constitutes a Delay in Puberty? Horm Res Pediatr 2014; 82 (4): 213-21.

[17] Hussein A, Farghaly H, Askar E, Metwalley K, Saad K, Zahran A et al. Etiological factors of short stature in children and adolescents: experience at a tertiary care hospital in Egypt. Ther Adv Endocrinol Metab. 2017; 8 (5): 75-80. 